\title{
ON SIMPSON'S QUADRATURE FORMULA FOR MAPPINGS OF BOUNDED VARIATION AND APPLICATIONS
}

\author{
SEVER SILVESTRU DRAGOMIR
}

\begin{abstract}
An estimation of remainder for Simpson's quadrature formula for mappings of bounded variation and applications in theory of special means (logarithmic mean, identric mean, etc..) are given.
\end{abstract}

\section{Introduction}

The following inequality is well known in the literature as Simpson's inequality:

$$
\mid \int_{a}^{b} f(x) d x-\frac{b-a}{3}\left[\frac{f(a)+f(b)}{2}+2 f\left(\frac{a+b)}{2}\right] \mid \leq \frac{1}{2880}\left\|f^{(4)}\right\|_{\infty}(b-a)^{5}\right.
$$

where the mapping $f:[a, b] \rightarrow R$ is supposed to be four time differentiable on the interval $(a, b)$ and having the fourth derivative bounded on $(a, b)$, that is

$$
\left\|f^{(4)}\right\|_{\infty}:=\sup _{x \in(a, b)}\left|f^{(4)}(x)\right|<\infty .
$$

Now, if we assume that $I_{h}: a=x_{0}<x_{1}<\cdots<x_{n-1}<x_{n}=b$ is a partition of the interval $[a, b]$ and $f$ is as above, then we have the Simpson's quadrature formula:

$$
\int_{a}^{b} f(x) d x=A_{S}\left(f, I_{h}\right)+R_{S}\left(f, I_{h}\right)
$$

where $A_{S}\left(f, I_{h}\right)$ is the Simpson's rule

$$
A_{S}\left(f, I_{h}\right)=: \frac{1}{6} \sum_{i=0}^{n-1}\left[f\left(x_{i}\right)+f\left(x_{i+1}\right)\right] h_{i}+\frac{2}{3} \sum_{i=0}^{n-1} f\left(\frac{x_{i}+x_{i+1}}{2}\right) h_{i}
$$

and the remainder term $R_{S}\left(f, I_{h}\right)$ satisfies the estimation

$$
\left|R_{S}\left(f, I_{h}\right)\right| \leq \frac{1}{2880}\left\|f^{(4)}\right\|_{\infty} \sum_{i=0}^{n-1} h_{i}^{5}
$$

Received May 28, 1998.

1991 Mathematics Subject Classification. 26D16, 26D99

Key words and phrases. Simpson's formula, special means. 
where $h_{i}:=x_{i+1}-x_{i}$ for $i=0, \ldots, n-1$.

When we have an equidistant partitioning of $[a, b]$ given by

$$
I_{n}: x_{i}:=a+\frac{b-a}{n} i, \quad i=0, \ldots, n
$$

then we have the formula

$$
\int_{a}^{b} f(x) d x=A_{S, n}(f)+R_{S, n}(f)
$$

where

$$
\begin{aligned}
A_{S, n}(f):= & \frac{b-a}{6 n} \sum_{i=0}^{n-1}\left[f\left(a+\frac{b-a}{n} i\right)+f\left(a+\frac{b-a}{n}(i+1)\right)\right] \\
& +\frac{2(b-a)}{3 n} \sum_{i=0}^{n-1} f\left(a+\frac{b-a}{n} \cdot \frac{2 i+1}{2}\right)
\end{aligned}
$$

and the remainder satisfies the estimation

$$
\left|R_{S, n}(f)\right| \leq \frac{1}{2880} \cdot \frac{(b-a)^{5}}{n^{4}}\left\|f^{(4)}\right\|_{\infty}
$$

For some other integral inqualities see the recent book [1].

\section{Simpson's Inequality for Mappings of Bounded Variation}

The following result holds:

Theorem 2.1. Let $f:[a, b] \rightarrow R$ be a mapping of bounded variation on $[a, b]$. Then we have the inequality

$$
\left|\int_{a}^{b} f(x) d x-\frac{b-a}{3}\left[\frac{f(a)+f(b)}{2}+2 f\left(\frac{a+b}{2}\right)\right]\right| \leq \frac{1}{3}(b-a) V_{a}^{b}(f)
$$

where $V_{a}^{b}(f)$ denotes the total variation of $f$ on the interval $[a, b]$.

Proof. Using the integration by parts formula for Riemann-Stieltjes integral we have:

$$
\int_{a}^{b} s(x) d f(x)=\frac{b-a}{3}\left[\frac{f(a)+f(b)}{2}+2 f\left(\frac{a+b}{2}\right)\right]-\int_{a}^{b} f(x) d x
$$

where

$$
s(x):=\left\{\begin{array}{ll}
x-\frac{5 a+b}{6}, & x \in\left[a, \frac{a+b}{2}\right) \\
x-\frac{a+5 b}{6}, & x \in\left[\frac{a+b}{2}, b\right]
\end{array} .\right.
$$


Indeed,

$$
\begin{aligned}
\int_{a}^{b} s(x) d f(x) & =\int_{a}^{\frac{a+b}{2}}\left(x-\frac{5 a+b}{6}\right) d f(x)+\int_{\frac{a+b}{2}}^{b}\left(x-\frac{a+5 b}{6}\right) d f(x) \\
& =\left[\left(x-\frac{5 a+b}{6}\right) f(x)\right]_{a}^{\frac{a+b}{2}}+\left[\left(x-\frac{a+5 b}{6}\right) f(x)\right]_{\frac{a+b}{2}}^{b}-\int_{a}^{b} f(x) d x \\
& \left.=\frac{b-a}{3}\left[\frac{f(a)+f(b)}{2}+2 f \frac{(a+b)}{2}\right)\right]-\int_{a}^{b} f(x) d x
\end{aligned}
$$

and the identity is proved.

Now, assume that $\Delta_{n}: a=x_{0}^{(n)}<x_{1}^{(n)}<\cdots<x_{n-1}^{(n)}<x_{n}^{(n)}=b$ is a sequence of divisions with $\nu\left(\Delta_{n}\right) \rightarrow 0$ as $n \rightarrow \infty$, where $\nu\left(\Delta_{n}\right):=\max _{i \in\{0, \ldots, n-1\}}\left(x_{i+1}^{(n)}-x_{i}^{(n)}\right)$ and $\xi_{i}^{(n)} \in\left[x_{i}^{(n)}, x_{i+1}^{(n)}\right]$.

If $p:[a, b] \rightarrow R$ is continuous on $[a, b]$ and $v:[a, b] \rightarrow R$ is of bounded variation on $[a, b]$, then

$$
\begin{aligned}
\left|\int_{a}^{b} p(x) d v(x)\right| & =\lim _{\nu\left(\Delta_{n}\right) \rightarrow 0} \sum_{i=0}^{n-1} p\left(\xi_{i}^{(n)}\right)\left[v\left(x_{i+1}^{(n)}-v\left(x_{i}^{(n)}\right)\right] \mid\right. \\
& \leq \lim _{\nu\left(\Delta_{n}\right) \rightarrow 0} \sum_{i=0}^{n-1}\left|p\left(\xi_{i}^{(n)}\right)\right|\left|v\left(x_{i+1}^{(n)}\right)-v\left(x_{i}^{(n)}\right)\right| \\
& \leq \max _{x \in[a, b]}|p(x)| \sup _{\Delta_{n}} \sum_{i=0}^{n-1}\left|v\left(x_{i+1}^{(n)}\right)-v\left(x_{i}^{(n)}\right)\right| \\
& =\max _{x \in[a, b]}|p(x)| V_{a}^{b}(v) .
\end{aligned}
$$

Applying the inequality (2.3) for $p(x)=s(x)$ and $v(x)=f(x)$ we get

$$
\left|\int_{a}^{b} s(x) d f(x)\right| \leq \max _{x \in[a, b]}|s(x)| V_{a}^{b}(f) .
$$

Taking into account the fact that the mapping $s$ is monotonous nondecreasing on the intervals $\left[a, \frac{a+b}{2}\right)$ and $\left[\frac{a+b}{2}, b\right]$ and

$$
s(a)=-\frac{b-a}{6}, \quad s\left(\frac{a+b}{2}-0\right)=\frac{1}{3}(b-a), \quad s\left(\frac{a+b}{2}\right)=-\frac{1}{3}(b-a)
$$

and $s(b)=\frac{b-a}{6}$ we deduce that $\max _{x \in[a, b]}|s(x)|=\frac{1}{3}(b-a)$. (2.1).

Now, using the inequality (2.4) and the identity (2.2) we deduce the desired result

Corollary 2.2. Suppose that $f:[a, b] \rightarrow R$ is a differentiable mapping whose derivative is integrable on $(a, b)$, i.e.,

$$
\left\|f^{\prime}\right\|_{1}:=\int_{a}^{b}\left|f^{\prime}(x)\right| d x<\infty .
$$


Then we have the inequality

$$
\left|\int_{a}^{b} f(x) d x-\frac{b-a}{3}\left[\frac{f(a)+f(b)}{2}+2 f\left(\frac{a+b}{2}\right)\right]\right| \leq \frac{1}{3}\left\|f^{\prime}\right\|_{1}(b-a) .
$$

The following corollary for Simpson's composite formula holds:

Corollary 2.3. Let $f:[a, b] \rightarrow R$ be of bounded variation on $[a, b]$ and $I_{h}$ a partition of $[a, b]$. Then we have the Simpson's quadrature formula (1.2) and the remainder term $R_{S}\left(f, I_{h}\right)$ satisfies the estimation:

$$
\left|R_{S}\left(f, I_{h}\right)\right| \leq \frac{1}{3} \gamma(h) V_{a}^{b}(f)
$$

where $\gamma(h):=\max \left\{h_{i} \mid i=0, \ldots, n-1\right\}$.

The case of equidistant partitioning is embodied in the following corollary:

Corollary 2.4. Let $I_{n}$ be an equidistant partitioning of $[a, b]$ and $f$ be as in Theorem 2.1. Then we have the formula (1.6) and the remainder satisfies the estimation:

$$
\left|R_{S, n}(f)\right| \leq \frac{1}{3 n}(b-a) V_{a}^{b}(f)
$$

Remark 2.5. If we want to approximate the integral $\int_{a}^{b} f(x) d x$ by Simpson's formula $A_{S, n}(f)$ with an accuracy less that $\varepsilon>0$, we need at least $n_{\varepsilon} \in N$ points for the division $I_{n}$, where

$$
n_{\varepsilon}:=\left[\frac{1}{3 \varepsilon} \cdot(b-a) V_{a}^{b}(f)\right]+1
$$

and $[r]$ denotes the integer part of $r \in R$.

Comments 2.6. If the mapping $f:[a, b] \rightarrow R$ is neither four time differentiable nor the fourth derivative in bounded on $(a, b)$, then we can not apply the classical estimation in Simpson's formula using the fourth derivative. But if we assurne that $f$ is of bounded variation, then we can use instead the formula (2.6).

We give here a class of mappings which are of bounded variation but having the fourth derivative unbounded on the given interval.

Let $f_{p}:[a, b] \rightarrow R, f_{p}(x):=(x-a)^{p}$ where $p \in(3,4)$. Then obviously

$$
f_{p}^{\prime}(x):=p(x-a)^{p-1}, \quad x \in(a, b)
$$

and

$$
f_{p}^{(4)}(x)=\frac{p(p-1)(p-2)(p-3)}{(x-a)^{4-p}}, \quad x \in(a, b) .
$$

It is clear that $f_{p}$ is of bounded variation and

$$
V_{a}^{b}(f)=(b-a)^{p}<\infty
$$


but $\lim _{x \rightarrow a+} f_{p}^{(4)}(x)=+\infty$.

\section{Applications for Special Means}

Let us recall the following means:

1. Arithmetic mean

$$
A=A(a, b):=\frac{a+b}{2}, \quad a, b \geq 0
$$

2. Geometric mean

$$
G=G(a, b):=\sqrt{a b}, \quad a, b \geq 0
$$

3. Harmonic mean

$$
H=H(a, b):=\frac{2}{\frac{1}{a}+\frac{1}{b}}, \quad a, b>0 ;
$$

4. Logarithmic mean

$$
L=L(a, b):=\frac{b-a}{\ln b-\ln a}, \quad a, b>0, a \neq b ;
$$

5. Identric mean

$$
I=I(a, b):=\frac{1}{e}\left(\frac{b^{b}}{a^{a}}\right)^{\frac{1}{b-a}}, \quad a, b>0, a \neq b ;
$$

6. p-Logarithmic mean

$$
L_{p}=L_{p}(a, b):=\left[\frac{b^{p+1}-a^{p+1}}{(p+1)(b-a)}\right]^{\frac{1}{p}}, p \in R \backslash\{-1,0\}, \quad a, b>0, a \neq b .
$$

It is well known that $L_{p}$ is monotonic nondecreasing over $p \in R$ with $L_{-1}:=L$ and $L_{0}:=I$. In particular, we have the following inequalities

$$
H \leq G \leq L \leq I \leq A
$$

In what follows, by the use of Theorem 2.1, we point out some new inequalities for the above means.

1. Let $f:[a, b] \rightarrow R(0<a<b), f(x)=x^{p}, p \in R \backslash\{-1,0\}$. Then

$$
\begin{aligned}
& \frac{1}{b-a} \int_{a}^{b} f(x) d x=L_{p}^{p}(a, b), \quad \frac{f(a)+f(b)}{2}=A\left(a^{p}, b^{p}\right), \\
& f\left(\frac{a+b)}{2}\right)=A^{p}(a, b),\left\|f^{\prime}\right\|_{1}=|p|(b-a) L_{p-1}^{p-1}, \quad p \in R \backslash\{-1,0,1\} .
\end{aligned}
$$

Using the inequality (2.5) we get

$$
\left|L_{p}^{p}(a, b)-\frac{1}{3} A\left(a^{p}, b^{p}\right)-\frac{2}{3} A^{p}(a, b)\right| \leq \frac{|p|}{3} L_{p-1}^{p-1}(b-a) .
$$


2. Let $f:[a, b] \rightarrow R(0<a<b), f(x)=\frac{1}{x}$. Then

$$
\begin{aligned}
& \frac{1}{b-a} \int_{a}^{b} f(x) d x=L^{-1}(a, b), \quad \frac{f(a)+f(b)}{2}=H^{-1}(a, b), \\
& f\left(\frac{a+b}{2}\right)=A^{-1}(a, b), \quad\left\|f^{\prime}\right\|_{1}=\frac{b-a}{G^{2}(a, b)} .
\end{aligned}
$$

Using the inequality (2.5) we get

$$
|3 A H-A L-2 H L| \leq \frac{(b-a)}{G^{2}} L H A .
$$

3. Let $f:[a, b] \rightarrow R(0<a<b), f(x)=\ln x$. Then

$$
\begin{aligned}
& \frac{1}{b-a} \int_{a}^{b} f(x) d x=\ln I(a, b), \quad \frac{f(a)+f(b)}{2}=\ln G(a, b) \\
& f\left(\frac{a+b}{2}\right)=\ln A(a, b), \quad\left\|f^{\prime}\right\|_{1}=\frac{b-a}{L(a, b)}
\end{aligned}
$$

Using the inequality (2.5) we get

$$
\left|\ln \left[\frac{I}{G^{1 / 3} A^{2 / 3}}\right]\right| \leq \frac{(b-a)}{3 L} .
$$

\section{References}

[1] D. S. Mitrinović, J. E. Pečarić and A. M. Fink, Inequalities for Functions and Their Integrals and Derivatives, Kluwer Academic Publishers, 1994.

Department of Computer and Math. Sci., Victoria University of Technology, PO Box 14428, Melbourne City, MC 8001, Victoria, Australia.

E-mail: sever@matilda.vut.edu.au 